\title{
Perturbation Study of Dissolution Apparatus Variables-A Design of Experiment Approach
}

\author{
Joseph Eaton ${ }^{1}$, Gang Deng, Walter W. Hauck, William Brown, \\ Ronald G. Manning, and Samir Wahab \\ US Pharmacopeia, 12601 Twinbrook Parkway, Rockville, MD 20852-1790, USA
}

\begin{abstract}
The specifications and acceptable ranges for nine critical dissolution apparatus variables were examined during a perturbation study of USP dissolution Apparatus 2 using USP Prednisone Reference Standard (RS) Tablets. A Design of Experiment (1) approach was used to screen the nine variables to determine the contribution of each, alone and in combination, to mean percent dissolved and standard deviation results. We observed a wide range of dissolution results, including several values that fell outside of current acceptance limits, even though the variables were kept within currently acceptable ranges. When we analyzed mean percent dissolved results, we found three variables that were statistically significant:level of deaeration, vessel type, and rotation speed. When we examined standard deviation results, we found that five variables or combinations of variables were statistically significant:vessel type, level of deaeration, paddle height, paddle height-vessel type interaction, and paddle height-level of deaeration interaction. We also found that the other variables examined - temperature, shaft wobble, vessel centering, vessel tilt, and base plate levelness - were not statistically significant within the ranges explored in this study. Acceptance ranges for several assembly variables may need to be more stringent or more precisely defined in order to decrease inter- and intralaboratory variability (reproducibility and repeatability) in dissolution testing.
\end{abstract}

\section{Introduction}

or nonsolution, orally administered dosage forms, the

- USP Performance test can rely on either disintegration

(General Chapter Disintegration $<701>$ ) or dissolution (General Chapter Dissolution <711>). The dissolution procedure is an important test both to establish bioequivalence and also for quality control. The procedure includes a complex sample preparation step in a dissolution apparatus. General Chapter $<711>$ specifies acceptance ranges for the dissolution vessel and variables of operation (2). During its multilaboratory studies of new Lot P Prednisone Reference Standard Tablets (Prednisone RS Tablets) used in periodic performance testing (PVT), USP noted substantial interlaboratory variability in results. This has led USP to conduct a comprehensive set of studies to examine potential assembly deviations that contribute to this variability. This study is one of a series of reports of these investigations (3-6). Based on these studies, USP may change statements in $\langle 711\rangle$ and allied documents to assist manufacturers in improving the USP Performance test when the dissolution procedure is chosen.

We designed this study to screen variables for Apparatus 2 (Paddle), with plans for a similar study of Apparatus 1 (basket) later on. Because this was a screening study, we did not examine exhaustively the effects of each variable but rather attempted to identify which variables were the most essential to control in order to achieve accurate and reproducible dissolution results. Previous research has focused on assembly variables such as dissolved gas, ves-

${ }^{1}$ Corresponding author. sel centering, vibration, vessel tilt, paddle height, rotation speed, base plate levelness, shaft wobble, temperature, vessel shape, belt tension, and shaft verticality (3,7-12). Building on these earlier studies, we attempted a more systematic approach that included an assessment of combination effects from two or more concurrently perturbed variables. In this study, nine variables were evaluated using

Table 1. Variables Included in Design of Experiment Study.

\begin{tabular}{|c|c|c|c|}
\hline Variable & Units & -1 Value & +1 Value \\
\hline Temperature & ${ }^{\circ} \mathrm{C}$ & 36.5 & 37.5 \\
\hline Shaft wobble & $\begin{array}{c}\mathrm{mm} \text { total } \\
\text { runout }\end{array}$ & 0.0 & 0.5 \\
\hline $\begin{array}{l}\text { Rotation } \\
\text { speed }\end{array}$ & rpm & 48.0 & 52.0 \\
\hline $\begin{array}{c}\text { Vessel } \\
\text { centering }\end{array}$ & $\begin{array}{c}\text { mm total } \\
\text { runout }\end{array}$ & 0.0 & 2.0 \\
\hline Vessel tilt & $\circ$ & 0.0 & 1.0 \\
\hline Paddle height & $\mathrm{mm}$ & 23.0 & 25.0 \\
\hline $\begin{array}{l}\text { Base plate } \\
\text { levelness }\end{array}$ & $\circ$ & 0.0 & 1.0 \\
\hline Vessel types & N/A & $\begin{array}{l}\text { Manufac- } \\
\text { turer's }\end{array}$ & Replacement \\
\hline $\begin{array}{c}\text { Level of } \\
\text { deaeration }\end{array}$ & N/A & $\begin{array}{c}\text { USP-deaer- } \\
\text { ated }\end{array}$ & $\begin{array}{c}\text { Non-deaer- } \\
\text { ated }\end{array}$ \\
\hline
\end{tabular}


a Design of Experiment approach:temperature, shaft wobble, rotation speed, vessel centering, vessel tilt, paddle height, base plate levelness, vessel type, and level of deaeration.

\section{Methods}

Vessel specifications and other aspects for mechanical calibration for a dissolution assembly and media are available to the dissolution chemist. General Chapter $<711>$ provides guidance for most of these. PhRMA (7) and ASTM (13) also provide acceptable ranges for the procedure.

\section{Perturbation Settings}

The perturbation settings used in this study were chosen to be near but within the upper or lower bound of ranges provided in USP, PhRMA, and ASTM references (Table 1).

\section{Temperature}

The dissolution medium temperature was maintained at $36.5^{\circ} \mathrm{C}$ or $37.5^{\circ} \mathrm{C}$ and was measured using an external thermometer placed inside the vessel.

\section{Shaft wobble}

For zero-wobble experiments, the paddle shafts supplied by the manufacturer were used. For $0.5-\mathrm{mm}$ wobble experiments, another set of shafts that had been previously bent by a machine shop were used. Wobble measurements were made using a wobble meter at a point approximately $1 \mathrm{~cm}$ above the paddle blade with the paddles raised above the top of the vessels. The entire head plate was then lowered to the appropriate height, maintaining the position of the shafts in the chuck.

\section{Rotation speed}

The speed was set to either $48.0 \mathrm{rpm}$ or $52.0 \mathrm{rpm}$ and confirmed using an external tachometer.

\section{Vessel centering}

Vessels were manually set to either $0 \mathrm{~mm}$ or $2 \mathrm{~mm}$ as measured using a centering device. The centering device was placed approximately $1 \mathrm{~cm}$ above the paddle blade, and the measurement was made inside the vessel with the paddle at $23 \mathrm{~mm}$ or $25 \mathrm{~mm}$.

\section{Vessel tilt}

Shims were inserted on the base plate underneath the vessel flange at the same position for all vessels to achieve a $1.0^{\circ}$ tilt as measured using a digital protractor on the top of the vessel. Shims were removed for $0^{\circ}$-tilt experiments.

\section{Paddle height}

The paddles were manually adjusted to $23 \mathrm{~mm}$ or $25 \mathrm{~mm}$ as measured by a depth gauge.

\section{Base plate levelness}

Shims were inserted under the leveling feet on one side of the dissolution tester to achieve a base plate levelness of $1.0^{\circ}$ as measured using a digital level. These were removed for zero-degree base plate levelness experiments.

\section{Vessel types}

The appropriate vessel types, manufacturer's or replacement, were used for each experiment.

\section{Deaeration}

The appropriate medium, USP deaerated or non-deaerated, was used for each experiment. A dissolved-gas meter capable of measuring total dissolved gas pressure (TDGP) and oxygen concentration was used to record these measurements.

\section{Dissolution}

Apparatus 2 dissolution experiments were performed using USP Prednisone RS Tablets Lot P0E203, six tablets per run. After $30 \mathrm{~min}$, samples were manually collected and filtered using a hydrophilic PVDF $0.45-\mu$ m syringe filter (Millex-HV, Millipore Corporation, Billerica, MA), discarding the first $5 \mathrm{~mL}$. Prednisone concentrations were determined using UV analysis at $242.0 \mathrm{~nm}$; percent dissolved values were calculated using the label claim of $10 \mathrm{mg}$. All experiments were conducted by the same analyst on the same dissolution assembly without provision of the manufacturer's name to preserve anonymity. All vessels and paddle shafts were serialized and kept in the same position for each experiment. All thermometers, timers, wobble meters, tachometers, levels, centering devices, depth gauges, and dissolved gas meters were within calibration. For USPdeaerated medium, Milli-Q water was heated to approximately $45^{\circ} \mathrm{C}$, vacuum filtered through a $0.45-\mu \mathrm{m}$ membrane filter, then vacuum degassed for an additional five minutes. For non-deaerated medium, Milli-Q water was heated to approximately $41^{\circ} \mathrm{C}$ and held at $41^{\circ} \mathrm{C}$ for at least $30 \mathrm{~min}$. The temperature was closely monitored to ensure a minimal equilibration time (less than $15 \mathrm{~min}$ ) for the dissolution medium inside the vessels. Manufacturer's vessels refer to the vessels supplied by the manufacturer of the dissolution tester. Replacement vessels refer to vessels manufactured by another company and sold as replacement vessels for the specific dissolution tester in use. Two responses were recorded for each experiment, the mean percent dissolved for the six positions of the test and the standard deviation of each mean percent dissolved value.

\section{Experimental Design}

Full-factorial designs involve a substantial number of experiments ( $2^{9}$ or 512 experiments in this case) and thus are not ideal for a screening process. With an incomplete factorial design, some effects are aliased with other effects. We thus chose an incomplete factorial design in which all main effects of each variable and all two-factor interactions were not aliased with each other. They were, however, aliased with three-factor and higher-order interactions, so we assumed those higher-order interactions were negligible enough that our choice of variables for further study 
Table 2. Experimental Design and Results.

\begin{tabular}{|c|c|c|c|c|c|c|c|c|c|c|c|}
\hline \multirow{2}{*}{ Run } & \multirow{2}{*}{ Temp } & \multirow{2}{*}{$\begin{array}{c}\text { Shaft } \\
\text { Wobble }\end{array}$} & \multirow{2}{*}{$\begin{array}{c}\text { Rotation } \\
\text { Speed }\end{array}$} & \multirow{2}{*}{$\begin{array}{c}\text { Vessel } \\
\text { Centering }\end{array}$} & \multirow{2}{*}{ Vessel Tilt } & \multirow{2}{*}{$\begin{array}{l}\text { Paddle } \\
\text { Height }\end{array}$} & \multirow{2}{*}{$\begin{array}{c}\text { Base } \\
\text { Plate } \\
\text { Levelness }\end{array}$} & \multirow{2}{*}{ Vessels } & \multirow{2}{*}{$\begin{array}{l}\text { Deaera- } \\
\text { tion }\end{array}$} & \multicolumn{2}{|c|}{ Percent Dissolved } \\
\hline & & & & & & & & & & Mean & SD \\
\hline$A^{1}$ & $37.0^{\circ}$ & - & 50.0 & - & - & + & - & - & - & 43.3 & 1.2 \\
\hline $\mathrm{B}^{1}$ & $37.0^{\circ}$ & - & 50.0 & - & - & + & - & - & - & 44.1 & 1.7 \\
\hline $\mathrm{C}^{1}$ & $37.0^{\circ}$ & - & 50.0 & - & - & + & - & - & - & 43.3 & 1.0 \\
\hline 1 & + & - & + & - & - & - & - & - & - & 46.3 & 1.5 \\
\hline 2 & - & + & - & - & + & - & + & + & + & 58.0 & 7.6 \\
\hline 3 & - & + & + & + & - & + & - & + & + & 87.6 & 0.7 \\
\hline 4 & + & + & - & - & + & - & - & - & + & 49.8 & 1.1 \\
\hline 5 & + & - & - & - & - & - & + & + & - & 48.1 & 8.7 \\
\hline 6 & - & - & - & - & - & - & - & - & - & 42.7 & 1.9 \\
\hline 7 & - & + & + & - & - & - & - & - & - & 46.4 & 2.3 \\
\hline 8 & - & - & + & - & + & + & - & - & - & 45.9 & 0.9 \\
\hline 9 & - & + & + & + & - & - & + & + & + & 74.8 & 6.6 \\
\hline 10 & - & + & + & + & + & - & - & + & - & 62.5 & 6.9 \\
\hline 11 & + & - & - & - & - & - & - & + & + & 70.6 & 9.8 \\
\hline 12 & + & + & + & + & + & - & + & - & - & 46.5 & 2.2 \\
\hline 13 & + & - & + & + & + & + & + & - & - & 48.1 & 1.6 \\
\hline 14 & - & - & + & - & - & - & - & + & - & 49.2 & 6.2 \\
\hline 15 & - & - & - & + & + & - & + & + & - & 48.7 & 8.5 \\
\hline 16 & - & - & + & + & - & + & + & - & - & 44.1 & 1.8 \\
\hline 17 & + & + & - & - & - & + & - & + & + & 56.4 & 7.8 \\
\hline 18 & + & - & + & - & + & - & + & + & + & 55.7 & 11.2 \\
\hline 19 & - & - & + & + & + & - & - & - & + & 58.5 & 2.1 \\
\hline 20 & - & - & + & - & + & - & + & - & - & 43.7 & 1.4 \\
\hline 21 & - & + & - & + & - & + & + & + & - & 42.6 & 1.5 \\
\hline 22 & + & + & + & - & - & - & + & - & + & 72.4 & 0.7 \\
\hline 23 & + & - & - & + & - & + & - & + & - & 43.7 & 1.0 \\
\hline 24 & - & - & - & - & + & + & + & + & - & 41.5 & 1.3 \\
\hline 25 & - & + & - & - & - & + & + & - & + & 68.4 & 5.4 \\
\hline 26 & - & - & + & - & - & + & + & - & + & 55.5 & 1.8 \\
\hline 27 & - & + & - & - & + & + & - & - & - & 42.6 & 1.5 \\
\hline 28 & + & + & + & + & - & - & - & + & - & 48.8 & 2.8 \\
\hline 29 & + & + & - & - & - & + & + & - & - & 43.2 & 1.1 \\
\hline 30 & + & + & + & + & - & + & + & - & + & 50.0 & 2.0 \\
\hline 31 & - & - & - & + & + & + & + & - & + & 48.8 & 1.9 \\
\hline 32 & + & - & - & + & - & + & - & - & + & 51.0 & 2.5 \\
\hline 33 & + & + & + & - & - & + & + & + & - & 45.2 & 1.4 \\
\hline 34 & + & + & - & + & + & - & - & + & + & 56.3 & 11.5 \\
\hline 35 & - & - & - & + & - & + & + & + & + & 55.4 & 2.5 \\
\hline 36 & + & + & - & + & + & + & + & + & - & 44.7 & 2.0 \\
\hline 37 & + & + & + & - & + & + & + & - & + & 56.9 & 2.8 \\
\hline 38 & - & - & - & - & + & + & - & + & + & 70.4 & 8.7 \\
\hline 39 & + & - & - & + & + & - & - & - & - & 42.1 & 1.2 \\
\hline
\end{tabular}


Table 2. Experimental Design and Results. (continued)

\begin{tabular}{|c|c|c|c|c|c|c|c|c|c|c|c|}
\hline \multirow{2}{*}{ Run } & \multirow{2}{*}{ Temp } & \multirow{2}{*}{$\begin{array}{c}\text { Shaft } \\
\text { Wobble }\end{array}$} & \multirow{2}{*}{$\begin{array}{c}\text { Rotation } \\
\text { Speed }\end{array}$} & \multirow{2}{*}{$\begin{array}{c}\text { Vessel } \\
\text { Centering }\end{array}$} & \multirow{2}{*}{ Vessel Tilt } & \multirow{2}{*}{$\begin{array}{l}\text { Paddle } \\
\text { Height }\end{array}$} & \multirow{2}{*}{\begin{tabular}{|c|} 
Base \\
Plate \\
Levelness
\end{tabular}} & \multirow{2}{*}{ Vessels } & \multirow{2}{*}{$\begin{array}{c}\text { Deaera- } \\
\text { tion }\end{array}$} & \multicolumn{2}{|c|}{ Percent Dissolved } \\
\hline & & & & & & & & & & Mean & SD \\
\hline 40 & + & + & + & + & + & + & - & - & - & 45.7 & 1.1 \\
\hline 41 & - & + & - & + & - & - & - & - & + & 54.7 & 2.9 \\
\hline 42 & - & - & + & + & + & + & + & + & + & 66.4 & 14.8 \\
\hline 43 & + & - & + & + & + & + & - & + & + & 74.6 & 4.6 \\
\hline 44 & + & - & - & + & - & - & + & - & + & 44.4 & 0.5 \\
\hline 45 & + & + & - & - & + & - & - & + & - & 44.5 & 2.9 \\
\hline 46 & - & + & + & - & + & - & - & + & + & 75.6 & 8.9 \\
\hline
\end{tabular}

${ }^{1} T$ These data are obtained under baseline, nonperturbed conditions.

was correct. A Design of Experiment statistical software package (Design-Expert Version 7.0.2, Stat-Ease, Inc., Minneapolis, MN) was employed to design a set of experiments that satisfied this condition for aliasing and then to analyze the data. The design with the smallest number of experiments that satisfied our aliasing requirement was a minimum run resolution $V$ design with the minimum number of experiments with two levels (denoted -1 and +1 ) of each of our nine factors (Table 1). (Resolution $V$ describes the level of aliasing; e.g., that the two-factor interactions are aliased with three-factor interactions.) Two responses were recorded: mean percent dissolved and standard deviation of each run of six tablets. The total number of experiments was 46 , and the order of experiments was randomized. Each factor was run an equal number of times, 23, at its -1 and +1 levels. Experiments were not replicated in this design, which made it imperative for scientists to accurately and precisely set each factor for each experiment. Data

\section{Table 3. Effects List for Mean Percent Dissolved Results.}

\begin{tabular}{|c|c|c|}
\hline Term & Effect* & \% Contribution \\
\hline Temperature & -4.5 & 1.1 \\
\hline Shaft wobble & 0.9 & 0.7 \\
\hline Rotation speed & 12.1 & 9.4 \\
\hline Vessel centering & -0.7 & 0.4 \\
\hline Vessel tilt & 1.6 & 0.0 \\
\hline Paddle height & -2.4 & 0.5 \\
\hline Base plate levelness & -2.2 & 1.2 \\
\hline Vessel type & 12.8 & 10.4 \\
\hline Level of deaeration & 31.0 & 52.3 \\
\hline All 2-factor interactions $(n=36)$ & N/A & 24.2 \\
\hline Total & N/A & 100.0 \\
\hline
\end{tabular}

*The effect size is the difference between the estimated average at the +1 setting minus that at the -1 setting (see Table 1) as a percent of the average at the -1 setting. analysis used a base 10 log transformation for both mean percent dissolved and standard deviation. Half-normal probability plots and the Shapiro-Wilk normality test were used to guide selection of the significant factors for both mean percent dissolved and standard deviation analyses. To illustrate the important variables, we present Pareto charts that show significance levels with (Bonferroni) and without adjustment for multiple testing. Because this was intended as a screening study, we did not adjust for multiple testing. Resulting $p$ values for all unselected factors were greater than 0.10 , and $p$ values for significant factors were all less than 0.05. An analysis of variance (partial sum of squares-type III) was performed for both mean and standard deviation of percent dissolved to quantify the contribution of each factor.

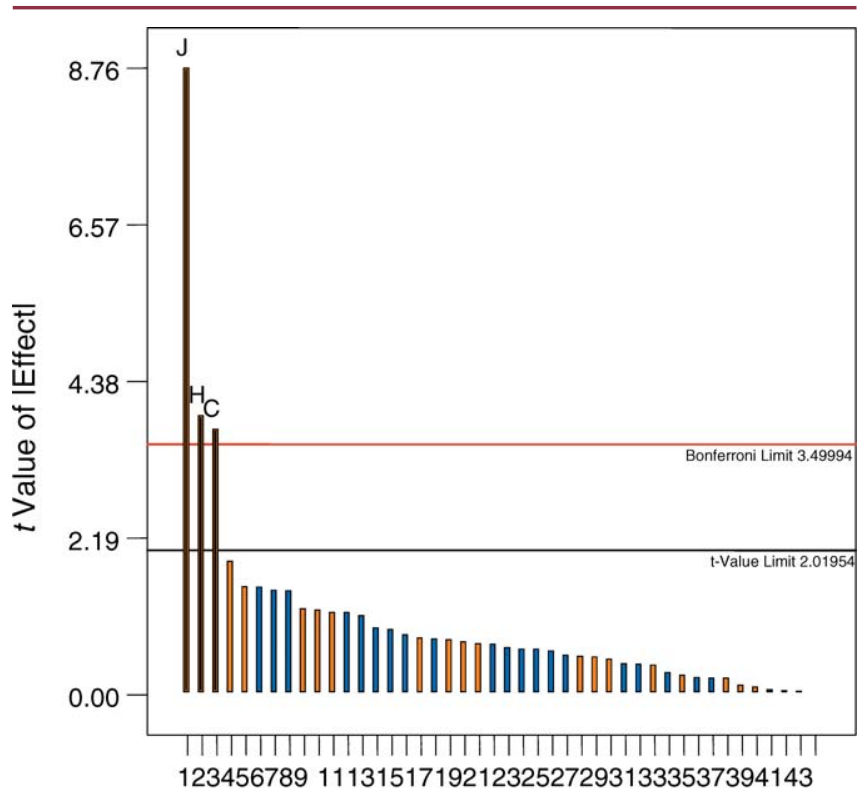

Rank

Figure 1. Pareto Chart for the analysis of mean percent dissolved showing the three significant variables: Level of Deaeration (J), Vessel Type (H), and Rotation Speed (C). 


\section{Results}

Experimental results demonstrate that combined perturbations affect both the mean percent dissolved values and the standard deviation values (Table 2). The baseline data obtained under nonperturbed conditions are listed at the top of the table. The 46 experiments performed under perturbed conditions, as indicated by $\mathrm{a}+$ or - for each variable, are listed next. Mean percent dissolved values ranged from $41.5 \%$ to $87.6 \%$, and standard deviation results ranged from 0.5 to 14.8 . Several experiments included values that fell outside of currently acceptable ranges (14).

\section{Mean Percent Dissolved Results}

Of the nine variables and 36 two-factor variables (Table 3 , Figure 1), three variables significantly affected mean percent dissolved: deaeration, vessel type, and rotation speed. A marginal effect due to temperature seems to be due to one observation (Run 3 in Table 2). When this data point is removed, temperature is not significantly affected by the specified perturbation (below the $t$-value limit) without affecting results for the other variables. All results are presented, therefore, with the data from run 3 omitted. The effects listed in Table 3 refer to the difference between the estimated average at the +1 setting minus that at the -1

Table 4. Effects List for Standard Deviation Results.

\begin{tabular}{|c|c|c|}
\hline Term & Effect* & $\%$ Contribution \\
\hline Temperature & -21.2 & 2.1 \\
\hline Shaft wobble & 17.5 & 0.0 \\
\hline Rotation speed & 11.8 & 0.1 \\
\hline Vessel centering & 18.7 & 0.0 \\
\hline Vessel tilt & 13.1 & 1.1 \\
\hline Paddle height & -27.1 & 3.5 \\
\hline $\begin{array}{l}\text { Base plate } \\
\text { levelness }\end{array}$ & -4.7 & 0.2 \\
\hline Vessel type & 182.1 & 37.7 \\
\hline Level of deaeration & 78.9 & 11.8 \\
\hline $\begin{array}{c}\text { Paddle } \\
\text { height/vessel type }\end{array}$ & -41.1 & 9.8 \\
\hline $\begin{array}{c}\text { Paddle } \\
\text { height/level of } \\
\text { deaeration }\end{array}$ & 61.3 & 8.0 \\
\hline $\begin{array}{l}\text { All other } 2 \text {-factor } \\
\text { interactions } \\
(n=34)\end{array}$ & N/A & 25.7 \\
\hline Total & $\mathrm{N} / \mathrm{A}$ & 100.0 \\
\hline
\end{tabular}

*The effect size is the difference between the estimated average at the +1 setting minus that at the -1 setting (see Table 1) as a percent of the average at the -1 setting. setting (see Table 1) as a percent of the average at the -1 setting. For example, when the rotation speed changes from $48.0 \mathrm{rpm}$ to $52.0 \mathrm{rpm}$, mean percent dissolved values increased by $12.1 \%$ (from $40.7 \%$ dissolved at $48.0 \mathrm{rpm}$ to $45.6 \%$ dissolved at $52.0 \mathrm{rpm})$.

\section{Standard Deviation Results}

Of the nine variables and 36 two-factor variables (Table 4, Figure 2), five variables significantly affected standard deviation results. Single-factor variables were vessel type, level of deaeration, and paddle height, and two-factor variables were paddle height-vessel type interaction and paddle height-level of deaeration interaction. Run 3 in Table 2 gave a standard deviation value that was identified to be an outlier. As noted above, reported results did not include this run. Inclusion of run 3 did not affect the results significantly. The effects listed in Table 4 were calculated as for Table 3.

\section{Discussion}

From many collaborative studies performed to determine the acceptance ranges for USP Prednisone RS Tablets (5), we have observed a high level of interlaboratory variability. Although a certain degree of interlaboratory variability is expected, it is possible that the dissolution test needs to be more precisely specified and performed in order to reduce this variability. One of the goals of this study was to identify apparatus variables that require more stringent acceptance criteria to achieve this type of specificity. We found six variables or combinations of variables that contributed significantly (mean and/or standard deviation) to dissolution results and five variables that did not.

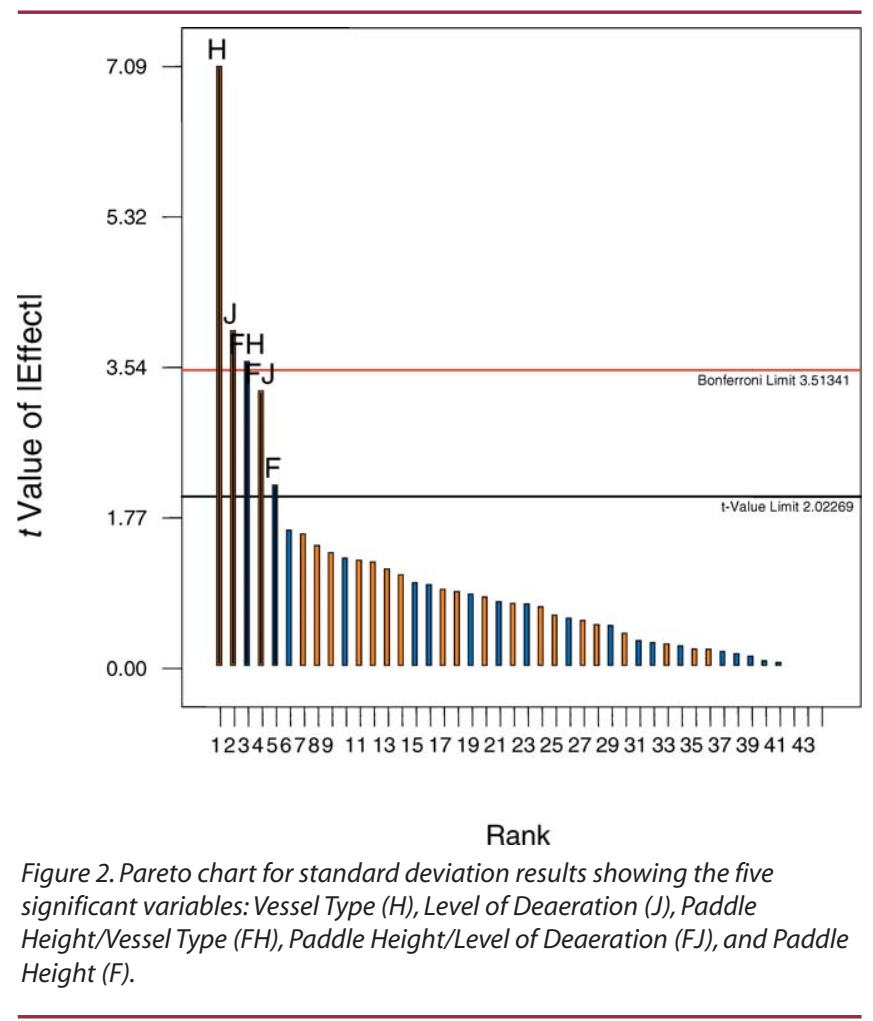




\section{Vessel Type}

Vessel type studied in two sets of six had a significant impact on both mean percent dissolved and standard deviation results. Acceptance criteria for vessel dimensions are specified in $<711>$. However, recent research has shown that geometric irregularities are found in dissolution vessels and affect dissolution results using USP Prednisone RS Tablets $(11,15)$. The two sets of vessels used in this study were similar in their geometric properties as measured using a 3-dimensional coordinate measuring machine (16). The replacement vessels were set arbitrarily to the high $(+1)$ value for the Design of Experiment study, not, however, because we believe them to be of inferior quality. This study shows that switching from one set of vessels to another, similar set of vessels leads to significant differences in both mean percent dissolved and standard deviation, suggesting that the current geometric acceptable ranges may not be appropriate.

\section{Rotation Speed}

We found that the current $\pm 4 \%$ rotation speed range appears to be too wide because mean percent dissolved of prednisone RS is significantly affected by rotation speed in this range. Continuous rotation speed monitoring is desirable. Further studies are needed to establish new acceptance limits.

\section{Paddle Height (Three Effects)}

Paddle height, both individually and as a component of two-factor interactions, was significant in the standard deviation analysis. The combination effect of paddle height on the standard deviation was greater for replacement vessels and non-deaerated medium. Equivalently, the combination effect of deaeration was more important at the $25-\mathrm{mm}$ height than at $23 \mathrm{~mm}$, and the difference between replacement and manufacturer's vessels was more pronounced at $23 \mathrm{~mm}$ than $25 \mathrm{~mm}$. Although these data may not be conclusive enough to warrant a tighter specification for paddle height, they do show that paddle height should be closely monitored (16). The height should be checked in conjunction with the vessel that will be used for the dissolution test using a gauge calibrated to be accurate. Heights should be periodically checked to ensure that no shafts have slipped out of their set positions.

\section{Deaeration}

Deaeration was the most significant variable for mean percent dissolved and the second most significant variable for standard deviation. The average oxygen concentration and total dissolved gas pressure (TDGP) for USP deaerated medium measured at the beginning of the experiment were $2.5 \pm 0.5 \mathrm{mg} / \mathrm{L}$ and $350 \pm 70 \mathrm{~mm} \mathrm{Hg}$, respectively. Values for non-deaerated medium were $6.8 \pm 0.7 \mathrm{mg} / \mathrm{L}$ and 620 $\pm 20 \mathrm{~mm} \mathrm{Hg}$, respectively. Validating any deaeration procedure against the oxygen concentration and TDGP values obtained for USP deaearated medium is crucial in obtaining valid dissolution results for USP Prednisone RS Tablets (3).

\section{Nonsignificant variables}

Several variables - temperature, shaft wobble, vessel centering, vessel tilt, and base plate levelness-did not significantly affect either mean percent dissolved or standard deviation results. This does not imply that these variables are unimportant in dissolution testing. It only suggests that in the ranges examined in this study, dissolution results were not significantly affected by the specified perturbations.

\section{Variability}

The pooled tablet-to-tablet standard deviation value for the 23 experiments using the manufacturer's vessels was 2.1. This is consistent with data from experiments under normal (nonperturbed) conditions that have shown relatively low variability using USP Prednisone RS Tablets (6). These findings may also help explain previous data that show low intralaboratory variability but high interlaboratory variability of prednisone dissolution results (5). Under most of the conditions studied, we obtained good precision, even when perturbations were present. Dissolution practitioners should not assume that apparently precise results alone correlate with true and accurate dissolution apparatus performance. Perturbations may be present in the system and may affect the mean value, which is generally increased by perturbations, but not the precision between the $6-8$ positions of the test.

\section{Conclusions}

By employing a Design of Experiment approach as a screening tool, we identified several variables that have a significant effect on mean percent dissolved and standard deviation results. Wide variation of results, including several that fell outside acceptance limits, were observed when variables were set to their upper and lower limits of currently acceptable ranges. Variables found to be significant-level of deaeration, vessel type (quality), rotation speed, and paddle height - may require more stringent acceptance criteria. Certain vessel attributes appear to be inadequately specified at this time and likely contribute to the high interlaboratory variability of prednisone dissolution results. Combination effects are present and make it more difficult to set appropriate mechanical calibration ranges. The ultimate goal is to determine acceptance criteria that will ensure acceptable accuracy and precision without unnecessarily tightening limits. USP will be conducting further experiments to identify these limits.

\section{References}

1. Montgomery, D. C. Design and Analysis of Experiments, $5^{\text {th }}$ ed.; John Wiley \& Sons, Inc.: New York, 2001.

2. Dissolution $<711>$. In United States Pharmacopeia and National Formulary USP 29-NF 24; The United States Pharmacopeial Convention, Inc.: Rockville, MD, 2006; pp 2675-2682.

3. Nithyananandan, P.;Deng, G.;Brown, W.; Manning, R.; Wahab, S. Evaluation of the Sensitivity of USP Pred- 
nisone Tablets to Dissolved Gas in the Dissolution Medium Using USP Apparatus 2. Dissolution Technol. 2006, 13 (3), 15-18.

4. The USP Council of Experts Biopharmaceutics Expert Committee, Dissolution Advisory Panel, and USP Staff. The USP Performance Verification Test, Part I:Proficiency Testing and the USP Dissolution Procedure. Submitted for publication, 2006.

5. Glasgow, M.;Dressman, S.;Brown,W.; Foster, T.;Schuber, S.; Manning, R.;Williams, R. L.;Hauck, W. W. The USP Performance Verification Test, Part II:Collaborative Study of USP's Lot P Prednisone Tablets. J. Pharm. Sci., in press.

6. Deng, G.; Ashley, A. J.; Brown,W. E.; Eaton, J.W.;Hauck,W. W.; Kikwai-Mutua, L. C.; Liddell, M. R.; Manning, R. G.; Munoz, J. M.; Nithyanandan, P.; Glasgow, M. J.;Stippler, E.;Wahab, S. Z.; Williams, R. L. The USP Performance Verification Test, Part III:USP Lot P Prednisone TabletsQuality Attributes and Experimental Variables Contributing to Dissolution Variance. Submitted for publication, 2006.

7. PhRMA Subcommittee on Dissolution Calibration. Dissolution Calibration: Recommendations for Reduced Chemical Testing and Enhanced Mechanical Calibration. Pharm. Forum 2000, 26 (4), 1149-1166.

8. Qureshi, S. A.; McGilveray, I. J.Typical Variability in Drug Dissolution Testing: Study with USP and FDA Calibrator Tablets and a Marketed Drug (Glibenclamide) Product. Eur.J.Pharm.Sci. 1999, 7,249-258.
9. Mirza,T.; Joshi, Y.; Liu, Q.;Vivilecchia, R. Evaluation of Dissolution Hydrodynamics in the USP, Peak, and Flat-Bottom Vessels Using Different Solubility Drugs. Dissolution Technol. 2005, 12 (1), 11-16.

10. Crist, B.; Spisak, D. Evaluation of Induced Variance of Physical Parameters on the Calibrated USP Dissolution Apparatus 1 and 2. Dissolution Technol. 2005, 12 (1),28-31.

11. Tanaka,M.; Fujiwara, H.;Fujiwara, M. Effect of Irregular Inner Shape of a Glass Vessel on Prednisone Dissolution Results. Dissolution Technol. 2005, 12 (4), 15-19.

12. Gao,Z.; Moore,T. W.;Doub,W. H.;Westenberger, B. J.; Buhse, L. F. Effects of Deaeration Methods on Dissolution Testing in Aqueous Media:A Study Using a Total Dissolved Gas Pressure Meter. J.Pharm.Sci. 2006, 95 (7), 1606-1613.

13. ASTM WK9936. New Standard Practice for Qualification of Basket and Paddle Dissolution Apparatus. ASTM International:West Conshohocken, PA.

14. United States Pharmacopeia. USP Prednisone Tablets RS Lot POE203, Disintegrating Tablets for Dissolution Performance Verification Test; Specification Sheet; United States Pharmacopeial Convention, Inc.: Rockville, MD.

15. Scott, P. Geometric Irregularities Common to the Dissolution Vessel. Dissolution Technol. 2005, 12 (1), 18-21.

16. Liddell,M.;Deng, G.;Hauck,W.W.;Brown,W. E.;Wahab, S. Z.; Manning, R. G. Evaluation of Glass Dissolution Vessel Dimensions and Irregularities. Dissolution Technol. 2007, $14(1), 28-33$.

\section{Now available on CD or DVD}

\section{Dissolution Technologies 1994-2003}

Get a six CD ROM set or one data DVD containing every issue of Dissolution Technologies from 1994 through 2003. The Table of Contents on the disks can be searched by Advertiser, Article and Issue. (The disks use Adobe Acrobat PDF files so you will need the free Adobe Acrobat Reader software.)

\section{Price: \$150 USA - \$170 outside USA Order online at www.dissolutiontech.com}

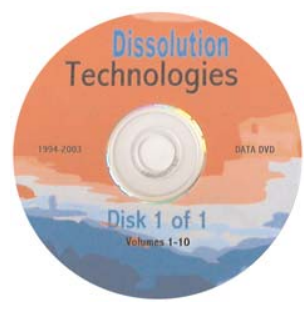

For additional ordering information contact:

Dissolution Technologies, Inc, attn: Sales/Back Issues Credit Card 9 Yorkridge Trail Hockessin, DE 19707 Phone (302) 235-0621; Fax (443) 946-1 264 Email: vgray@dissolutiontech.com

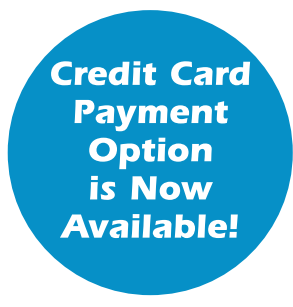

